\title{
XMM-Newton observation of the BAL Quasar PHL 5200:
}

\section{The big surprise}

\author{
W. Brinkmann ${ }^{1}$, E. Ferrero ${ }^{2}$, and M. Gliozzi ${ }^{3}$ \\ 1 Centre for Interdisciplinary Plasma Science, Max-Planck-Institut für Extraterrestrische Physik, Postfach 1312, \\ 85741 Garching, Germany \\ 2 Max-Planck-Institut für Extraterrestrische Physik, Postfach 1312, 85741 Garching, Germany \\ 3 George Mason University, Dept. of Phys. and Astron., MS 3F3, 4400 University Dr., Fairfax, VA 22030-4444, \\ USA
}

Received 30 January 2002 / Accepted 21 February 2002

\begin{abstract}
XMM-Newton observations of the BAL quasar PHL 5200 revealed that the X-ray emission attributed to the quasar originates from a nearby, optically unidentified radio source. Although visible in X-rays as well the flux from PHL 5200 is by a factor $\gtrsim 20$ smaller than previously anticipated. Thus most of the scenarios for a physical interpretation of the BAL phenomenon, based on the apparently unexpected X-ray properties of this quasar have to be revisited. We further discuss the properties of the optically very faint radio source.
\end{abstract}

Key words. galaxies: active - quasars - X-rays: galaxies

\section{Introduction}

Broad Absorption Line quasars (BALQSOs) have optical/UV spectra showing deep, wide absorption troughs, extending up to $\Delta v \sim 0.1-0.2 c$, displaced to the blue of their corresponding emission lines. BALQSOs constitute about $10-15 \%$ of optically selected QSOs and they are generally found only among the radio quiet population (Stocke et al. 1992) although recently, the first radioloud BALQSOs have been detected (Becker et al. 1997; Brotherton et al. 1998). The BAL phenomenon is suspected to result from a line of sight passing through highly ionized, high column density $\left(\log N_{\mathrm{H}}>20 \mathrm{~cm}^{-2}\right)$ absorbers, flowing outward from the nuclear region with high velocities. Recent work suggests that BALQSOs are normal, radio quiet quasars (RQQ) seen from an unusual direction (Weyman et al. 1991; Hamann et al. 1993), i.e., orientation is indeed the cause of the BAL phenomenon and all radio-quiet QSOs have BAL regions.

Most of the problems with an early model in which many clouds intercept the ionizing radiation and re - radiate it in emission lines are overcome by the disk wind picture of Murray et al. (1995). In that model the lineemitting gas emerges from the disk, there is no cloud confinement problem, the wind naturally produces the smooth line profiles and the covering fraction of the wind is $10 \%$, which explains the observed fraction of $\mathrm{BAL}$ quasars.

Send offprint requests to: W. Brinkmann, e-mail: wpb@rzg.mpg.de
In a similar, but more general model for the disk wind emission from quasars (Elvis 2000) the BAL region is not confined to the equatorial region of the quasars, but to intermediate regions when the wind, rising vertically from the disk gets bent radially under the influence of the radiation pressure from the quasar continuum. Here as well, the solid angle covered by the outflow $(\sim 10 \%)$ determines the fraction of BAL quasars.

An alternative interpretation is that BALQSO are, in analogy to Narrow Line Seyfert Galaxies, in an early evolutionary phase of high accretion rate. Strong support for this scenario comes from a principal component analysis of optical data of quasars by Boroson (2002) who finds that BALQSO occupy a phase space region characterized by high accretion rates and close to Eddington optical luminosities. For the X-ray spectra one then expects intrinsic power laws significantly steeper than in normal QSO, and some examples of steep spectra might exist (PHL 5200: Mathur et al. 2001, PG 1411+442: Wang et al. 1999). An evolutionary scheme is further preferred by observations of radio-loud BAL quasars, found in follow-up observations of the FIRST radio survey (Becker et al. 2000) which do not support the scenario that BAL quasars are all normal quasars seen edge-on, contrary to the popular orientation model of radio-loud quasars.

Thus, BALQSOs provide a unique probe for studying the evolutionary state and physical conditions near the nucleus of most QSOs. X-ray data, i.e., the amount of absorption, the depth and position of absorption edges - and the simultaneous exploitation of the UV and Xray spectra (Mathur et al. 1994; Wang et al. 1999) can 
provide important constraints for the physically allowed parameter ranges of the BAL material.

Until recently, the X-ray properties of BALQSOs as a class were unknown. In a study of BALQSOs from publicly available data of deep pointed ROSAT observations Green \& Mathur (1996) mainly found upper limits for the sources and it is now commonly believed that the weakness of the $\mathrm{X}$-ray flux from BALQSO is to be related to the fact that the emission regions are seen through heavy attenuation (Goodrich 1998).

Due to its higher energy bandpass $(2-10 \mathrm{keV})$ ASCA was more suited to study objects with high intrinsic absorption. However, many of the optically brightest BALQSO remained undetected in long ASCA observations (cf., Brinkmann et al. 1999; Gallagher et al. 1999) and for others limited photon statistics precluded a definite spectral diagnosis. In a recent Chandra survey of a sample of 10 bright BALQSO eight sources were detected (Green et al. 2001) and a simultaneous fit to the combined data supported the picture that BALQSO are similar to radio-quiet objects with extra intrinsic absorption. Further, in an analysis of a sample of eight quasars with broad UV absorption lines, observed by ASCA and Chandra, Gallagher et al. (2001) find an average power law slope of $\Gamma \approx 2.0$ and signatures of significant intrinsic absorption.

PHL 5200 is the prototype of a small subgroup of BALQSOs showing deep absorption with relatively low radial velocities (Turnshek et al. 1988; Goodrich 1997). Although it is thus perhaps not a prototypical BALQSO it provided for quite some time the best detection of X-ray emission from such objects. Mathur et al. (1996) have observed the BALQSO PHL5200 with ASCA and found it to be strongly intrinsically absorbed $\left(N_{\mathrm{H}} \sim 10^{23} \mathrm{~cm}^{-2}\right)$. As it is rather distant $(z=1.98)$ only a total of $\sim 1000$ counts were collected in all four instruments which inhibited a detailed spectral analysis.

In a second $\sim 85 \mathrm{ks}$ observation with ASCA in November 1999 Mathur et al. (2001) obtained sufficient photon statistics for more accurate spectral fits. The surprising result was that the hard power law index of PHL 5200 was steep, with photon index $\Gamma \sim 2.5$ and that the strong absorber with $N_{\mathrm{H}}=5 \times 10^{23} \mathrm{~cm}^{-2}$ does not cover the source completely. These results seem to support the hypothesis that BALQSOs represent an early evolutionary state of quasars (Mathur 2000) and, further, the proposed analogy to NLS1 galaxies. The fact that the soft $\mathrm{X}$-ray spectrum is only partially covered by the absorber ( $\sim 90 \%)$ suggests a complex physical absorber, possibly with multiple lines of sights. In a re-analysis of the ASCA data, applying corrections for the degradation of the SIS detectors, Gallagher et al. (2001) find a flatter power law slope, however with large errors, of $\Gamma=2.17_{-0.47}^{+0.60}$.

As the X-ray data from PHL 5200 serve as one of the touchstones for models of BAL quasars we tried to improve the data quality of this object utilizing XMMNewton's superior sensitivity and spatial resolution as well as its broader bandpass and spectral capabilities. In the next section we will describe the XMM-Newton observation of PHL 5200. We will then discuss PHL 5200 itself, then the nearby bright source and comment on the scientific implications for BALQSO research. We will limit this work to the immediate surroundings of PHL 5200 and defer the analysis of the X-ray rich XMM-Newton field of view to a forthcoming paper.

\section{The XMM-Newton observation}

PHL 5200 was observed by XMM-Newton on May 28, 2001 for a total of $\sim 46 \mathrm{ks}$. The EPIC PN was in Full Window mode with a thick filter; the MOS cameras in Full Window mode as well.

Figure 1 shows a Gaussian smoothed $\sim 7.5^{\prime} \times 7.5^{\prime}$ field around the source position from the PN camera. All single and double events with "flag $=0$ " were taken; no energy selection was performed. Clearly visible is the structure of the PN chips (the nominal pointing position is on CCDNR $=4$ ).

The most surprising result is that PHL 5200 is the faint object indicated by a cross, not the bright source to the north-west. This means, the spectral results deduced from previous ASCA observations (Mathur et al. 1995; Mathur et al. 2001) can not be attributed to PHL 5200 but to the brighter, previously not identified object. As a matter of fact Mathur et al. (1995) note that the object they identify with PHL 5200 is $\sim 1$ ' 1 away from the nominal position of the quasar but they attribute this discrepancy to the pointing uncertainties of ASCA.

A comparison of the ASCA count rates with the XMMNewton data of the bright object, using the spectral information given in Mathur et al. (2001) yields excellent agreement between the two measurements. This confirms the association of the ASCA source with the bright XMM object and rules out any dramatic temporal variations of the quasar itself. The X-ray position found for PHL 5200 is $\alpha=22^{\mathrm{h}} 28^{\mathrm{m}} 30.24$ and $\delta=-05^{\circ} 18^{\prime} 54^{\prime \prime} .5$ (J2000); the position of the unknown source is $\alpha=22^{\mathrm{h}} 28^{\mathrm{m}} 26.43$, $\delta=-05^{\circ} 18^{\prime} 21^{\prime \prime} .5$. There is no prominent optical object at this position; the attempts to determine the properties of the faint optical counterpart candidates are described in an accompanying paper (Wagner et al. 2002).

\section{PHL 5200}

We determined the $0.5-10 \mathrm{keV}$ X-ray flux from PHL 5200 by counting the photons in a circular region around the quasar position with radius $r=15^{\prime \prime}$. This small extraction radius was chosen to avoid contamination from the nearby bright object. The count rate was then corrected using the encircled energy function given by Aschenbach (2001). The background counts were obtained from the source free region on the same CCD chip.

We thus obtained from the $\mathrm{PN}$ in a net observation time of $39242 \mathrm{~s}$ a total of 76 counts, yielding a count rate of $1.94 \times 10^{-3} \mathrm{cts} / \mathrm{s}$. For the MOS1 we get 29 counts in $45345 \mathrm{~s}$, for the MOS2 41 counts in $45367 \mathrm{~s}$, resulting in 
count rates of $6.4 \times 10^{-4} \mathrm{cts} / \mathrm{s}$ and $9.1 \times 10^{-4} \mathrm{cts} / \mathrm{s}$, respectively. Unfortunately, the small number of measured photons does not provide any reliable spectral information. Therefore, for the conversion of count rates into flux, we assume that the source spectrum can be described by a power law with photon index $\Gamma=2$. The most critical parameter for this conversion is the amount of absorption towards the source. Assuming Galactic absorption $\left(N_{\mathrm{H}}=5.26 \times 10^{20} \mathrm{~cm}^{-2}\right)$, or values of $10^{22} \mathrm{~cm}^{-2}$, and $10^{23} \mathrm{~cm}^{-2}$, we obtain un-absorbed $0.5-10 \mathrm{keV}$ fluxes of $6.3 \times 10^{-15} \mathrm{erg} \mathrm{cm}^{-2} \mathrm{~s}^{-1}, 1.9 \times 10^{-14} \mathrm{erg} \mathrm{cm}^{-2} \mathrm{~s}^{-1}$, and $7.3 \times 10^{-14} \mathrm{erg} \mathrm{cm}^{-2} \mathrm{~s}^{-1}$, respectively. Here, we have taken the count rates from the PN camera which are consistent within the statistical errors with the MOS rates.

With these fluxes the K-corrected X-ray luminosity of PHL 5200 would be in the range $L_{0.5-10 \mathrm{keV}}=$ $(1.7-19.4) \times 10^{44} \mathrm{erg} \mathrm{s}^{-1}$, using a Friedman cosmology with $H_{0}=50 \mathrm{~km} \mathrm{~s}^{-1} \mathrm{Mpc}^{-1}$ and $q_{0}=0.5$. Thus PHL 5200 is in X-rays in any case a rather low-luminosity quasar, althoughit is optically very bright $\left(M_{\mathrm{B}}=-27.4\right)$. Using the luminosity at $2 \mathrm{keV}$ the X-ray loudness, $\alpha_{\mathrm{ox}}=$ $-0.384 \log \left(L_{2 \mathrm{keV}} / L_{2500 \mathrm{~A}}\right)$ is $\alpha_{\mathrm{ox}} \sim 2.2$ for the case of Galactic absorption. For $N_{\mathrm{H}}=10^{23} \mathrm{~cm}^{-2}$ the value would be 1.78 and for even higher absorption PHL 5200 would have an X-ray loudness like ordinary radio-quiet quasars (Yuan et al. 1998). However, an X-ray spectrum with considerably better photon statistics will be necessary to determine reliably the spectral slope and the column density of the absorbing matter.

\section{The radio source}

PHL 5200 is radio quiet, with $\mathrm{S}_{5 \mathrm{GHz}}<0.8$ mJy ( $5 \sigma$ upper limit, Stocke et al. 1984). All of the NED "identifications" with strong sources in the Green Bank and Parkes surveys are incorrect.

The cataloged radio object $4 \mathrm{C}-05.93$ (Scheuer \& Wills 1966; Munro 1971), with an integrated flux density of $1.1 \pm 0.2 \mathrm{Jy}$ at $327 \mathrm{MHz}$ is $\sim 1$ ' 1 from the position of PHL 5200. We have overlaid the $1465 \mathrm{MHz}$ contour lines of this triple source (Gopal-Krishna \& Sramek 1980) on the X-ray image in Fig. 1. The position of the weak unresolved component between the two lobes (Comp B) is coincident with the center position of the X-ray source. About half of the total radio flux, unseen in the lowresolution VLA observation, arises from a powerful bridge joining the two outer components (Gopal-Krishna 1978). The source has a very steep radio spectrum at low frequencies $\left(\alpha_{178-408 \mathrm{MHz}}=-1.8 \pm 0.4\right)$ which flattens at higher frequencies to $\alpha=-0.85 \pm 0.05$. This spectral behavior, the prominent radio bridge between the two lobes, and the large misalignment angle of $28^{\circ}$ between the lines joining the central component to the peaks in the outer lobes are reminiscent of radio sources evolving in a dense cluster medium. As no optical counterpart brighter than the POSS plate limit could be found at the position of the central source Gopal-Krishna \& Sramek (1980) argue that

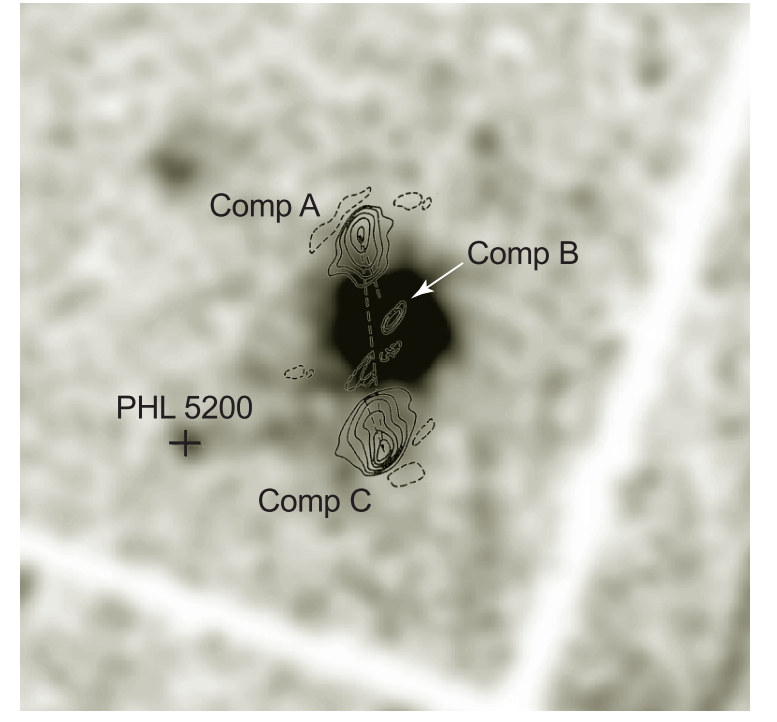

Fig. 1. Gaussian smoothed PN image of an $\sim 7.5^{\prime} \times 7.5^{\prime}$ sky region around PHL 5200, overlaid with the $1465 \mathrm{MHz}$ VLA radio contours. The position of the quasar is marked by a cross.

the source lies in a distant cluster with $z \gtrsim 0.3$, and thus the quasar may be seen through the core of this cluster.

\section{XMMU J222826.7-051821}

The X-ray source appears to be not point like but slightly extended. Its radial count rate profile is definitely wider than the encircled energy function measured for the instruments (Ghizzardi \& Molendi 2001) but the overall signal to noise is perhaps too low for a rigorous spatial analysis.

The ASCA observations of this object indicate a complex spectral form and the published best fit consisted of a power law model with high energy cutoff and a partially covering absorber (Mathur et al. 2001).

Using this model and limiting the fit to the sensitive energy band of the ASCA detectors $(\sim 0.7-8 \mathrm{keV})$ we obtain for the PN data an acceptable fit, however with slightly different parameters as found from ASCA. Our best fit parameters are $\Gamma=1.14 \pm 0.77, N_{\mathrm{H}}=$ $(1.42 \pm 0.33) \times 10^{22} \mathrm{~cm}^{-2}$, covering fraction $77 \pm 14 \%$ and a cutoff energy $E_{\mathrm{c}}=6.6 \pm 8.1 \mathrm{keV}$. A simple power law with partially covering absorber resulted in an equally good fit, however with steeper power law slope $(\Gamma=1.72 \pm 0.155)$ and higher covering fraction of $84.9 \pm 3.8 \%$.

In Fig. 2 we plot the XMM data and the corresponding best fit model for the $0.7-8 \mathrm{keV}$ range. The model/data ratio shows strong deviations between model and data outside the fitted energy range. At low energies we see a clear excess of photons over the model and at energies $>9 \mathrm{keV}$ there is a strong indication for a line structure. Illegal variable name.

The data quality is, however, perhaps not sufficient for a very detailed spectral analysis. Only about 4200 photons were collected in total from the three instruments and the source seems to be embedded in a region of weaker 


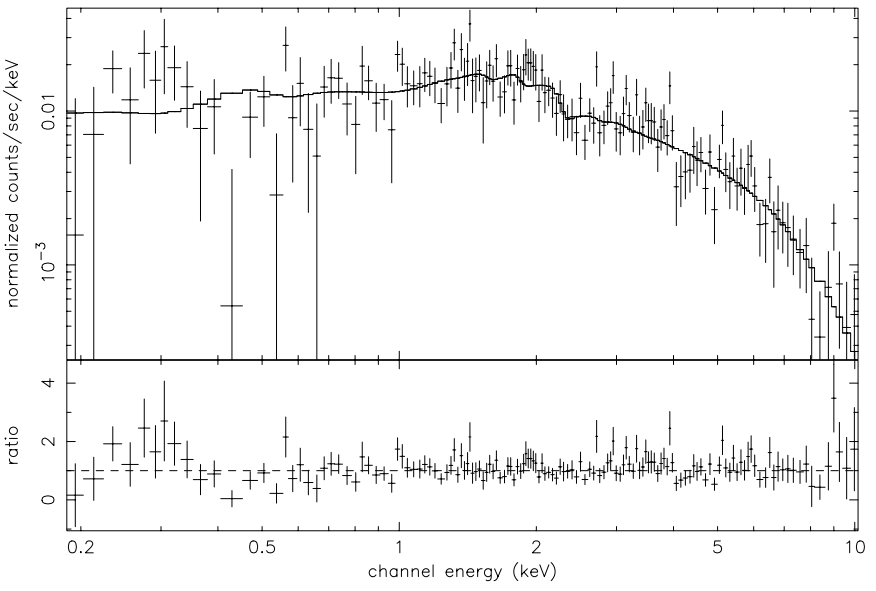

Fig. 2. Fit of a power law with exponential cutoff plus a partially covering absorber to the PN data. The fit was limited to the $0.7-8.0 \mathrm{keV}$ energy range. The model/data ratio at the bottom shows clear deviations of the model from the data outside this energy range.

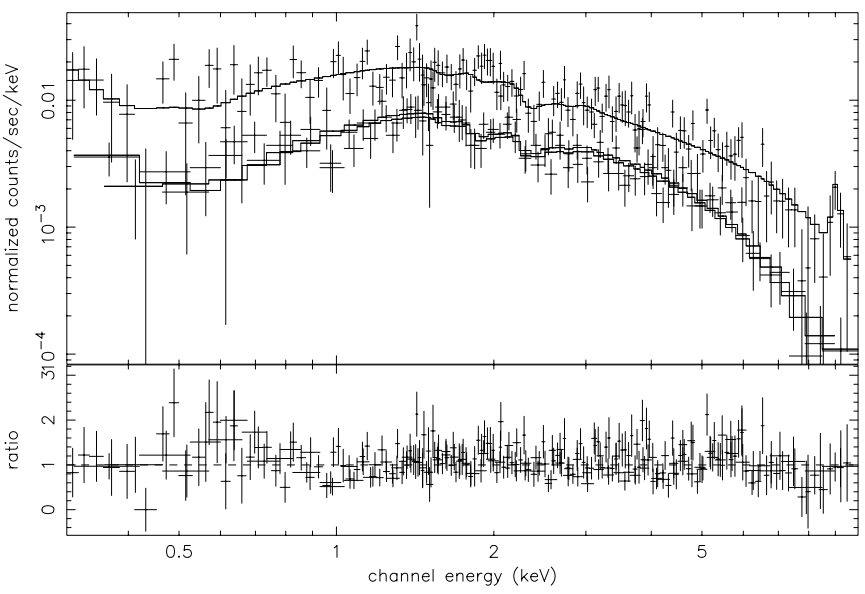

Fig. 3. Fit of a broken power law plus a soft black body and a Gaussian line to the combined PN and MOS data. For details see text.

emission, extending to the south-west (see Fig. 1). The excess at low energies is certainly real but the residuals differ between the MOS and PN cameras. The line structure near $9 \mathrm{keV}$ is not visible in any of the PN chips, it occurs exclusively from the source region.

The best fit to the whole range of the PN plus combined MOS data $\left(\chi_{\text {red }}^{2}=0.95\right.$ for 139 d.o.f. $)$ was obtained with a broken power law $\left(\Gamma_{1}=0.47 \pm 0.06, \Gamma_{\mathrm{h}}=\right.$ $1.68 \pm 0.13, E_{\mathrm{b}}=2.98 \pm 0.19 \mathrm{keV}$; these parameters are then fixed for the subsequent fit), an additional black body component $\left(T_{\mathrm{bb}}=0.02 \pm 0.001 \mathrm{keV}\right)$ and a Gaussian line $\left(E_{\text {line }}=9.03 \pm 0.12 \mathrm{keV}\right)$. The absorbing column density was fixed to the Galactic value. The strong bump in the residuals around $0.6 \mathrm{keV}$ demonstrates the remaining uncertainties in the instrument's cross calibration.

\section{Conclusions}

An XMM-Newton EPIC observation of the BAL quasar PHL 5200 revealed that the X-ray flux attributed to the quasar actually originates from the nearby radio source $4 \mathrm{C}-05.93$.

PHL 5200 itself is a rather low-luminosity object unless the value of an intrinsic absorption column density is exceedingly high. In that case its intrinsic X-ray loudness $\alpha_{\text {ox }}$ would be quite normal for a radio-quiet quasar. The spectral shape of PHL 5200 could not be determined due to the low signal. Models for BAL QSOs based on the anticipated "unusual" X-ray properties of PHL 5200 have to be reconsidered.

The nature of the radio source $4 \mathrm{C}-05.93$ remains unclear. The best fit spectral model for the XMM data must only be regarded as a parameterization of the spectral slope and can hardly be used for a physical interpretation. A curved spectrum plus an additional soft component is in agreement with Galactic absorption only. The data seem to require the presence of a high energy emission line, but the statistical significance (about 20 photons in the line region) is far from compelling. The optical data (Wagner et al. 2002) do not constrain the physical properties of the source either and the scenario of a radio galaxy in a cluster, as proposed from the radio data, gets support from the slight extent of the X-ray source and the weak extended X-ray emission towards the south-west of the object. However, the X-ray spectrum does not show clear indications for emission from these individual components.

A definite determination of the $\mathrm{X}$-ray properties of this object require additional data for a better signal to noise ratio as well as a reliable cross-calibration between the different EPIC instruments.

Acknowledgements. This research has made use of the NASA/IPAC Extragalactic Data Base (NED) which is operated by the Jet Propulsion Laboratory, California Institute of Technology, under contract with NASA. This work is based on observations with XMM-Newton, an ESA science mission with instruments and contributions directly funded by ESA Member States and the USA (NASA).

\section{References}

Aschenbach, B. 2001, Proc. SPIE, 4496 [astro-ph/0109375]

Becker, R. H., Gregg, M. D., Hook, I. M., et al. 1997, ApJ, 479, L93

Becker, R. H., White, R. L., Gregg, M. D., et al. 2000, ApJ, 538, 72

Boroson, T. A. 2002, ApJ, 565, 78

Brinkmann, W., Wang, T. G., Matsuoka, M., \& Yuan, W. 1999, A\&A, 345, 43

Brotherton, M. S., van Breugel, W., Smith, R. J., et al. 1998, ApJ, 505, L7

Elvis, M. 2000, ApJ, 545, 63

Gallagher, S. C., Brandt, W. N., Sambruna, R. M., \& Mathur, S. 1999, ApJ, 519, 549

Gallagher, S. C., Brandt, W. N., Chartas, G., \& Garmire, G. P. 2001, ApJ, in press [astro-ph/0110579] 
Ghizzardi, S., \& Molendi, S. 2001, Proc. of the conference New Visions of the X-ray Universe, ESTEC Nov. 2001

Goodrich, R. W. 1997, ApJ, 474, 606

Goodrich, R. W. 1998, in Proc. of the 18th Texas Symp., ed.

A. Olinto, J. A. Frieman, \& D. N. Schramm (World Sci. Publ., Singapore), 222

Gopal-Krishna 1978, Bull. Astron. Soc. India, 6, 98

Gopal-Krishna, \& Sramek, R. A. 1980, A\&A, 90, L1

Green, P. J., \& Mathur, S. 1996, ApJ, 462, 637

Green, P. J., Aldcroft, T. L., Mathur, S., Wilkes, B. J., \& Elvis, M. 2001, ApJ, in press [astro-ph/0105258]

Hamann, F., Korista, K. T., \& Morris, S. L. 1993, ApJ, 415, 541

Mathur, S. 2000, MNAS, 314, L17

Mathur, S., Wilkes, B., \& Fiore, F. 1994, ApJ, 434, 493

Mathur, S., Elvis, M., \& Wilkes, B. 1995, ApJ, 452, 230

Mathur, S., Matt, G., Green, P. J., Elvis, M., \& Singh, K. P. 2001, ApJ, 551, 13
Munro, R. E. B. 1971, Aust. J. Phys., 24, 263

Murray, N., Chiang, J., Grossman, S. A., \& Voit, G. M. 1995, ApJ, 451, 498

Scheuer, P. A. G., \& Wills, D. 1966, ApJ, 143, 274

Stocke, J. T., Foltz, C. B., Weymann, R. J., \& Christiansen, W. A. 1984, ApJ, 280, 476

Stocke, J. T., Morris, S. L., Weyman, R. J., \& Foltz, C. B. 1992, ApJ, 396, 48

Turnshek, D. A., Grillmair, C. J., Foltz, C. B., \& Weymann, R. J. 1988, ApJ, 325, 651

Wang, T. G., Wang, J. X., Brinkmann, W., \& Matsuoka, M. 1999, ApJ, 519, L35

Wagner, S., Heidt, J., \& Tapken, Ch. 2002, A\&A, submitted

Weyman, R. J., Morris, S. L., Foltz, C. B., \& Hewett, P. C. 1991, ApJ, 373, 23

Yuan, W., Brinkmann, W., Siebert, J., \& Voges, W. 1998, A\&A, 330, 108 